\title{
Safety Encoding in the Basal Amygdala
}

\author{
Susan Sangha, ${ }^{1}$ James Z. Chadick, ${ }^{1}$ and Patricia H. Janak ${ }^{1,2,3}$ \\ ${ }^{1}$ Ernest Gallo Clinic and Research Center, University of California at San Francisco, Emeryville, California 94608, and ${ }^{2}$ Wheeler Center for the Neurobiology \\ of Addiction, and ${ }^{3}$ Department of Neurology, University of California, San Francisco, California 94158
}

Learning to fear and avoid life-threatening stimuli are critical survival skills but are maladaptive when they persist in the absence of a direct threat. Thus, it is important to detect when a situation is safe and to increase behaviors leading to naturally rewarding actions, such as feeding and mating. It is unclear how the brain distinguishes between dangerous and safe situations. Here, we present a novel protocol designed to investigate the processing of cues that predict danger, safety, or reward (sucrose). In vivo single unit recordings were obtained in the basal amygdala of freely behaving rats undergoing simultaneous reward, fear, and safety conditioning. We observed a population of neurons that did not respond to a Fear Cue but did change their firing rate during the combined presentation of a fear cue simultaneous with a second, safety, cue; this combination of Fear + Safety Cues signified "no shock." This neural population consisted of two subpopulations: neurons that responded to the Fear + Safety Cue but not the Fear or Reward Cue ("safety" neurons), and neurons that responded to the Fear + Safety and Reward Cue but not the Fear Cue ("safety + reward" neurons). These data demonstrate the presence of neurons in the basal amygdala that are selectively responsive to Safety Cues. Furthermore, these data suggest that safety and reward learning use overlapping mechanisms in the basal amygdala.

\section{Introduction}

Post-traumatic stress disorder (PTSD) is associated with a loss of stimulus discrimination, resulting in generalized fear responses to nonthreatening stimuli (van der Kolk, 1997). While generalized fear expedites an individual's ability to respond quickly to a stimulus, it also causes overreaction to nonthreatening stimuli. Therefore, accurately classifying stimuli as dangerous or safe is important in initiating the proper emotional response. The mechanisms of learning to distinguish between dangerous and safe stimuli and to flexibly switch behavior accordingly can be investigated through safety conditioning, a form of Pavlovian conditioned inhibition (for review, Christianson et al., 2012). During safety conditioning, a cue signifying no aversive outcome becomes a learned safety signal, whereas a cue predicting something aversive becomes a learned danger signal. Impaired fear inhibition to safety cues, resulting in overgeneralized fear, is associated with PTSD (Jovanovic et al., 2010, Jovanovic et al., 2012).

The amygdala is important for mediating emotional responses and related memories (for review, Ledoux, 2000, Baxter and Murray, 2002, Pape and Paré, 2010, Salzman and Fusi, 2010). The human amygdala tracks the predictive aversive value of stimuli as they reverse from fear to safety (Schiller et

Received July 11, 2012; revised Dec. 5, 2012; accepted Dec. 26, 2012.

Author contributions: S.S. and P.H.J. designed research; S.S. performed research; S.S. and J.Z.C. analyzed data; S.S. and P.H.J. wrote the paper.

This work was supported by funding from the State of California for Medical Research on Alcohol and Substance Abuse through the University of California at San Francisco (P.H.J.). We thank Rebecca Reese and Irene Grossrubatscher for technical assistance, and Dr. T. Michael Gill for programming and recording guidance.

Correspondence should be addressed to either Susan Sangha or Patricia H. Janak, Ernest Gallo Clinic and Research Center, University of California at San Francisco, 5858 Horton Street, Suite 200, Emeryville, CA 94608. E-mail: susansangha@gmail.com or pjanak@gallo.ucsf.edu.

DOI:10.1523/JNEUROSCI.3302-12.2013

Copyright $\odot 2013$ the authors $\quad 0270-6474 / 13 / 333744-08 \$ 15.00 / 0$ al., 2008). Interestingly, as animals learn that a shockpredictive cue no longer predicts shock during behavioral extinction, neural responses selective for the extinguished cue emerge in the basal amygdala (BA) (Herry et al., 2008). This may reflect a type of safety encoding, as the fear cue comes to signal "no shock." Further, the BA contains neurons that respond differentially to fear and reward cues (Muramoto et al., 1993; Paton et al., 2006; Shabel and Janak, 2009; Shabel et al., 2011). Collectively, this suggests that the BA may contain neurons that are responsive to safety cues, although it is not clear how the representation of safety cues might differ from fear or reward cues.

Evidence suggests safety learning mechanisms overlap with reward learning (Rescorla, 1969a; Walasek et al., 1995; Tanimoto et al., 2004; Rogan et al., 2005; Leknes et al., 2011). For example, learned safety can act as a behavioral antidepressant in mice (Pollak et al. 2008), and a safety signal can produce a conditioned place preference in mice (Rogan et al., 2005). However, no studies have directly compared BA neural processing during safety and reward learning. To address this, we developed a protocol to investigate fear, reward, and safety learning in parallel, where we recorded activity of BA single units in rats as they switched their behavior in response to conditioned cues resulting in freezing, inhibition of freezing, or reward seeking. We found that rat BA neurons that respond to the Fear + Safety Cue, but not the Fear Cue, providing evidence that single neurons can respond selectively to a cue signifying safety. Furthermore, a subset of these neurons showed cue-evoked changes in firing rate in the same direction to both the Fear + Safety Cue and the Reward Cue. These data support our hypotheses that: (1) subpopulations of BA neurons are selective for safety cues; and (2) safety and reward cue processing involve overlapping circuitry. 


\section{Materials and Methods}

Subjects. Long Evans male rats (Harlan) weighing 350-400 g at the beginning of experiments were single housed under a $12 \mathrm{~h}$ light/dark cycle (lights on 07:00) and handled for 1 week before commencing experiments. All procedures were performed during the light cycle and approved by the Gallo Center Institutional Animal Care and Use Committee in accordance with the National Institute of Health guidelines. Rats had ad libitum access to food and water up until the third reward learning session, at which point they were restricted to $22 \mathrm{~g}$ of food per day for the remainder of the experiment.

Behavioral apparatus. The experimental chambers, used in all experiments and obtained from Med Associates, were Plexiglas boxes $(32 \mathrm{~cm}$ length $\times 31 \mathrm{~cm}$ width $\times 35 \mathrm{~cm}$ height) encased in sound-attenuating shells. A recessed port $3 \mathrm{~cm}$ above the floor and located in the center of one wall was used to deliver sucrose. Entries and exits into and out of this port were monitored via an infrared beam. Two lights $(28 \mathrm{~V}, 100 \mathrm{~mA})$ located $12 \mathrm{~cm}$ from the floor on the wall opposite the port provided constant illumination. A light $(28 \mathrm{~V}, 100 \mathrm{~mA})$ located $33 \mathrm{~cm}$ above the floor on the wall opposite the port served as the $20 \mathrm{~s}$ continuous light cue. A high-frequency "tweeter" speaker (ENV-224BM) located $25 \mathrm{~cm}$ from the floor on the wall opposite the port was used to deliver the auditory cues. Footshock was delivered through a grid floor via a constant current aversive stimulator (ENV-414S). A video camera located at the top of the sound-attenuating shell recorded the rat's behavior for offline video analysis.

Reward, fear, and safety training. The three cues signifying Reward, Fear or Safety were a $20 \mathrm{~s}$ continuous $3 \mathrm{kHz}$ tone $(70 \mathrm{~dB})$, a $20 \mathrm{~s}$ pulsing 11 $\mathrm{kHz}$ tone (200 ms on, $200 \mathrm{~ms}$ off; $70 \mathrm{~dB})$, or a $20 \mathrm{~s}$ continuous light $(28 \mathrm{~V}$, $100 \mathrm{~mA}$ ) counterbalanced across subjects, with the caveat that the light cue was reserved for the Safety Cue in most subjects. The light served as the Fear Cue in 3 of the 8 rats represented in Figures $1 B-D, 2$ of the 8 rats represented in Figure 2, and 2 of the 14 rats implanted with electrodes. Acquisition of fear did not differ between rats that received footshocks paired with the tone cue $(n=23)$ versus the light cue $(n=7)$ (two-way repeated-measures ANOVA, $\left.F_{(3,75)}=1.504, p>0.05\right)$. In Phase 1 of training (Fig. 1A; six sessions), a $20 \mathrm{~s}$ Reward Cue was followed by $3 \mathrm{~s}$ delivery of a $10 \%$ sucrose solution $(100 \mu \mathrm{l})$ into a port accessible to the rat (3 s sucrose delivery commenced pseudorandomly between 10 and $20 \mathrm{~s}$ after Reward Cue onset for 25 trials; ITI, 90-130 s, where ITI is intertrial interval). Phase 2 of training consisted of one session of habituation to the future Fear Cue and Safety Cue during a session in which Reward Cue training continued ( 25 reward trials; ITI, 90-130 s). The future Fear Cue and Safety Cue were presented separately 5 times each for $20 \mathrm{~s}$ without reinforcement to allow subjects to habituate to their presentation, thereby reducing any baseline freezing to these novel cues. In Phase 3, safety learning commenced (four sessions), while Reward Cue training continued ( $3 \mathrm{~s}$ sucrose delivery commenced $18 \mathrm{~s}$ after Reward Cue onset; 15 trials), along with the additional presentation of the $20 \mathrm{~s}$ Fear Cue followed by a mild $0.5 \mathrm{~s}$ footshock at the offset of the Fear Cue $(0.4 \mathrm{~mA} ; 4$ trials). In separate trials, this same $20 \mathrm{~s}$ Fear Cue was paired with a $20 \mathrm{~s}$ Safety Cue resulting in no footshock (15 trials). Trials in which the $20 \mathrm{~s}$ Safety Cue was presented alone without any footshock were also included (10 trials) to assess whether any freezing developed to the Safety Cue as a result of being paired with the Fear Cue as well as providing the animal with additional trials that contained a Safety Cue-no shock contingency. Trials were presented pseudorandomly (ITI, 100-140 s).

Retardation of acquisition training. A separate group of rats was trained as in the previous paragraph with two modifications. First, after the habituation session and before the first safety session there was a separate fear conditioning session in which the $20 \mathrm{~s}$ Fear Cue was followed by a $0.5 \mathrm{~s}, 0.4 \mathrm{~mA}$ footshock (four trials; ITI, 3-5 min). The second modification was to reduce the number of Fear Cue-shock trials during each safety session from four trials to three. Both protocols still resulted in each rat receiving a total of 16 Fear Cue-shock trials by the end of the last safety session. After the last safety session a test for retardation of acquisition followed: 1 day after the last safety session, the Safety Cue was followed by a $0.5 \mathrm{~s}, 0.4 \mathrm{~mA}$ footshock (one session, four trials; ITI, 3-5 $\mathrm{min})$. Freezing to the Safety Cue was measured $1 \mathrm{~d}$ later.
Behavioral analyses. Fear behavior was assessed offline from videos by measuring freezing, defined as complete immobility with the exception of respiratory movements, which is an innate defensive behavior (Blanchard and Blanchard, 1969; Fendt and Fanselow, 1999). The total time spent freezing was quantified during the entire $20 \mathrm{~s}$ of each cue presentation. Reward behavior was assessed by calculating the percentage of trials in which the rat entered the port where sucrose was delivered during the presentation of the Reward Cue. Behavioral data were analyzed using one-way or two-way repeated-measures ANOVA, followed by Tukey's post hoc test when indicated by significant $(p<0.05)$ main effects or interactions.

Surgery. Rats were anesthetized with isoflurane and stereotaxically implanted bilaterally with fixed 8-electrode arrays (NeuroBiological Laboratories) directed at the BA (relative to bregma: anteroposterior, -2.04 to $-2.92 \mathrm{~mm}$ posterior; mediolateral, 4.1 to $4.9 \mathrm{~mm}$; dorsoventral, 6.6 to $7.5 \mathrm{~mm}$ ventral from brain surface (Paxinos and Watson, 2007; Fig. 3A). Rats were allowed 7-10 d to recover in which they had ad libitum access to food and water.

In vivo single unit recordings. Neuronal activity was recorded with commercial hardware and software, including headstage amplifiers and programmable amplifiers, filters $(0.4$ and $5 \mathrm{kHz})$, and multichannel spike-sorting software (Plexon). Implanted rats were connected to the recording apparatus via a swivel commutator. Discrimination of individual units was performed offline by using principal component analysis of waveform shape. Single cells were identified by constancy of waveform shape, cross-correlograms, and interspike intervals (Janak, 2002). In addition, quantitative J3 and Davies-Bouldin validity index (DB) statistics were calculated. High J3 values and low DB values are indicative of good single unit isolation (Davies and Bouldin, 1979; Nicolelis et al., 2003; Herry et al., 2008). Stability of units across sessions was assessed by calculating principal component space cylinders using WaveTracker (Plexon). In addition, linear correlation values between time-shifted average waveforms were calculated (Jackson and Fetz, 2007; Herry et al., 2008). As a control, the $r$ values from average waveforms of randomly paired neurons and sessions were computed. Only units deemed stable across sessions using these procedures were included in the analysis. To increase statistical power, the data for the last two safety sessions were combined.

Classification of neurons. For each neuron, significance of cue-evoked firing rates was determined using a 10,000 round paired permutation test (Hesterberg et al., 2005) comparing the averaged 20 s precue baseline period to the first $200 \mathrm{~ms}$ after cue onset during the last two safety sessions. That is, the $20 \mathrm{~s}$ precue baseline firing rates and the $200 \mathrm{~ms}$ postcue firing rates for a given cue were shuffled and redistributed independently 10,000 times. The differences between the baseline and postcue firing for the single real case and the 10,000 reshuffled cases were used to create a distribution. In accordance with the permutation test, if the actual mean difference was in the $<2.5 \%$ of either tail, it was considered significant. The $p$ values were then adjusted for multiple corrections using the Benjamini-Hochberg correction with a corrected cutoff of 0.05 (Benjamini and Hochberg, 1995). Neurons with a significant change in firing rate to both the Fear and Fear + Safety Cues were classified as "SAME"; i.e., increase in firing rate to both cues or decrease in firing rate to both cues. Neurons with a significant change in firing rate to the Fear but not the Fear + Safety Cues or vice versa were classified as "SELECTIVE." Neurons without a significant change in firing rate to either the Fear or Fear + Safety cues were classified as "NO CHANGE." To avoid false positives, neurons that showed a significant cue-evoked inhibition using this permutation test were only included in the final analyses if the baseline firing frequency was $>0.05 \mathrm{~Hz}$ in at least six of the eight Fear trials.

To directly compare cue-evoked responding of a single unit to two different cues, a similar analysis using a 10,000 round, two-sample permutation test was conducted independently comparing the firing rate activity within each of four $50 \mathrm{~ms}$ bins after the onset of each cue. If the actual mean difference in responding between two cues was in the $<2.5 \%$ of either tail of the distribution created by 10,000 rounds of reshuffling, it was considered significant. The $p$ values were then adjusted for multiple corrections using the Benjamini-Hochberg correction with a corrected cutoff of 0.05 (Benjamini and Hochberg, 1995). 
A

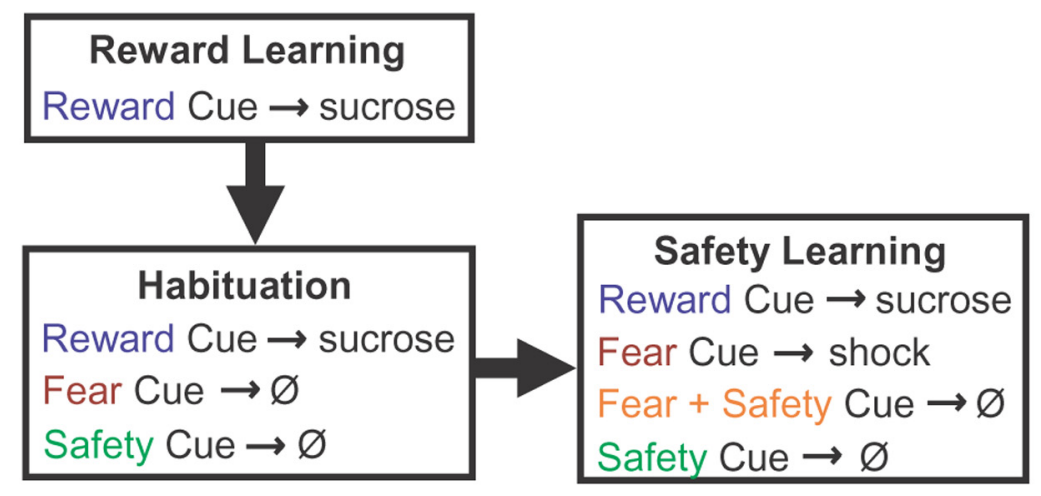

B

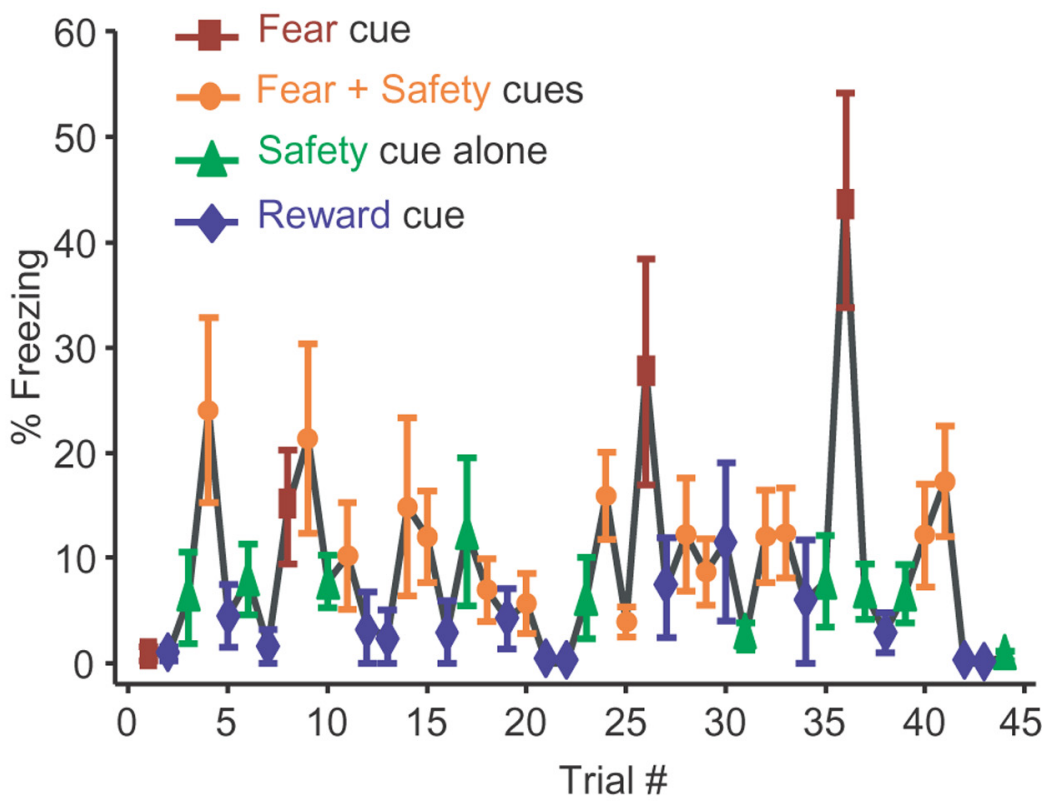

C

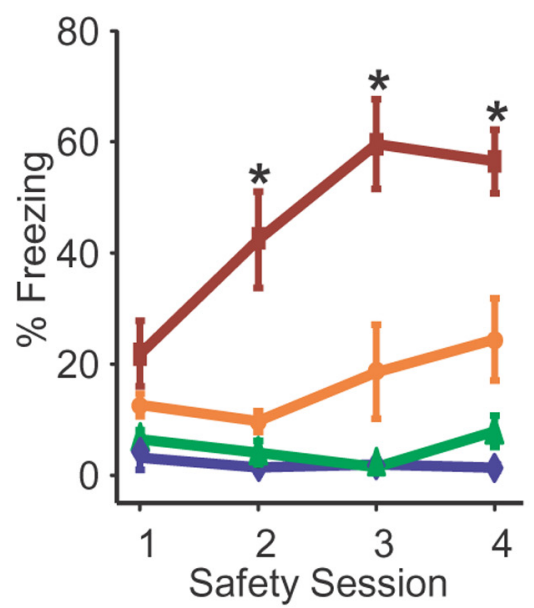

D

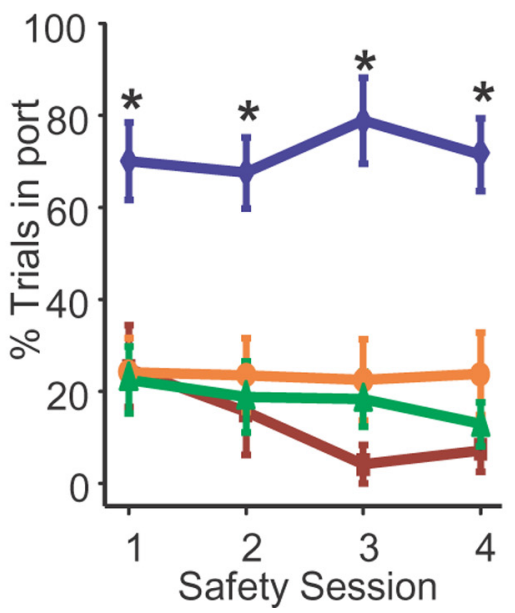

Figure 1. Decreased freezing in the presence of the safety cue. A, Protocol for reward, fear, and safety training. During reward learning, a Reward (ue was followed by delivery of sucrose solution into a port accessible to the rat (six sessions). In habituation (one session), Reward Cue training continued. In addition, the future Fear Cue and Safety Cue were presented without reinforcement. During safety learning (four sessions), Reward Cue training continued and the Fear Cue was followed by a footshock. On separate trials this same Fear Cue was paired with a Safety Cue, resulting in no footshock. Trials in which the Safety Cue was presented alone without any footshock were also included. Trials were presented pseudorandomly. $\boldsymbol{B}$, Mean ( \pm SEM) freezing response during each trial in the first safety session, indicated by percentage of time spent freezing during each cue type. By the end of the first safety session, animals $(n=8)$ distinguished between Fear and combined Fear + Safety Cues. C, Greater freezing

Histology. Rats were deeply anesthetized with sodium pentobarbital. A 10 s, $19 \mu$ A direct current was passed through each wire to mark each electrode tip. Rats were then perfused with formalin containing 3\% potassium ferrocyanide. Sections $(50 \mu \mathrm{M})$ were stained against acetylcholinesterase, and only units recorded from electrode wires verified to be in the BA were included in the analyses (Fig. 3A).

\section{Results}

\section{Decreased freezing in the presence of} the safety cue

To determine whether presentation of a Safety Cue significantly suppressed fear behavior within a behavioral session also containing Fear and Reward Cue presentations, we examined the behavior of rats $(n=8)$ within behavioral sessions that contained presentations of all three cue types (Fig. 1A; see Materials and Methods). A one-way repeated-measures ANOVA was used to test for freezing differences across the 44 trials of the first of four safety sessions (Fig. 1B). Freezing differed significantly across the 44 trials $\left(F_{(7,301)}=3.615, p<\right.$ 0.001). Most notably, Tukey post hoc comparisons of the last Fear trial to each subsequent trial indicated that freezing was significantly higher $(p<0.05)$ during the last Fear trial than any of the subsequent Reward, Fear + Safety, and Safety alone trials of the first safety session. Thus, subjects began to distinguish between Fear and Safety cues by the end of the first of four safety sessions.

Differential behavioral responding to the Fear Cue continued to increase over subsequent safety conditioning sessions, as depicted in Figure 1C. A two-way repeatedmeasures ANOVA revealed a significant interaction between safety session and cue type $\left(F_{(9,45)}=5.877, p<0.001\right)$ and a main effect of cue type $\left(F_{(3,15)}=37.899, p<\right.$ $0.001)$. The percent time spent freezing was significantly greater during the Fear Cue than during the Fear + Safety Cues, Safety Cue alone, or Reward Cue for safety sessions 2, 3 and 4 (post hoc Tukey's, $p<0.001$ ). Thus, freezing was significantly reduced in the presence of the Safety Cue. Also, importantly, very little freezing was observed when the Safety Cue was presented alone, showing the Safety Cue

during the Fear Cue. Mean ( \pm SEM) freezing response during each safety session for each cue type; ${ }^{*} p<0.05$ as compared to mean freezing response to Reward, Safety, and Fear + Safety Cues within each session. $D$, Reward seeking is specific to the Reward Cue. Mean ( \pm SEM) reward-seeking (port entry) behavior during each safety session for each cue type; ${ }^{*} p<0.05$ as compared to mean reward-seeking behavior to Fear, Safety, and Fear + Safety Cues within each session. 

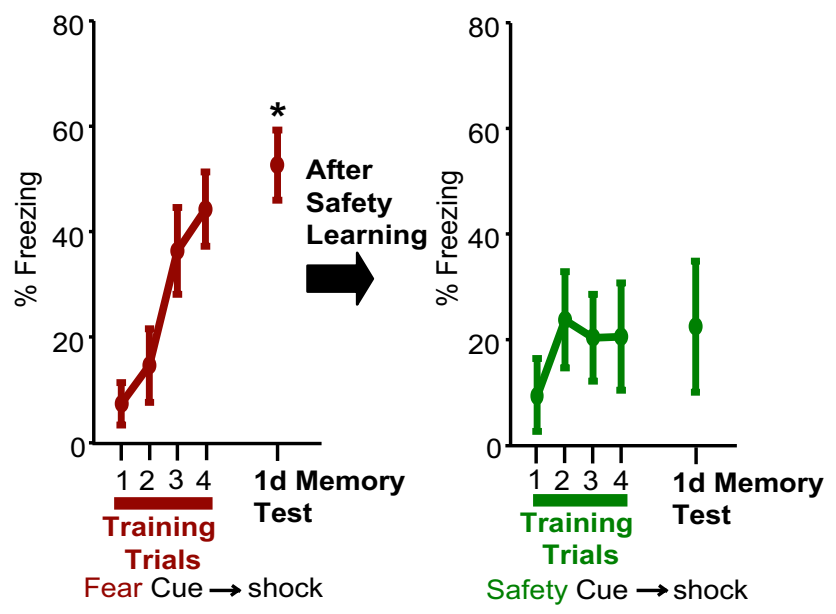

Figure 2. Retardation of acquisition to the Safety Cue. Mean ( \pm SEM) freezing responses for eight rats during each of the Fear Cues during fear conditioning are shown in the left panel for the four training trials and the memory test $1 \mathrm{~d}$ later. Freezing during the $1 \mathrm{~d}$ memory test was significantly higher than that during the first training trial $\left({ }^{*} p<0.05\right)$. All rats then underwent safety conditioning. One day after the last safety session, the Safety Cue was paired with a footshock four times (right panel). Presentation of the Safety Cue did not elicit significant freezing either during training or during the $1 \mathrm{~d}$ memory test.

did not acquire any fear-inducing qualities by being paired with the Fear Cue.

Differential responding to the Reward Cue was also observed. Figure $1 D$ shows the averaged reward-seeking behavior as measured by the percentage of trials in which the animal entered the port where sucrose was delivered during cue presentations, and during each safety session for each cue type. A two-way repeatedmeasures ANOVA revealed a significant main effect of cue type $\left(F_{(3,15)}=19.318, p<0.001\right)$. The percentage of trials in which the rats entered the port was significantly greater during the Reward Cue than during any other cue type across all sessions (post hoc Tukey's, $p<0.001$ ).

These findings indicate that this behavioral protocol successfully produces conditional reward seeking in response to a Reward Cue, high freezing to a Fear Cue, and decreased freezing to the Fear Cue in the presence of a Safety Cue. Since the Safety Cue reduces the freezing response normally elicited by the Fear Cue, the Safety Cue can be considered a conditioned inhibitor (Rescorla, 1969b).

\section{Retardation of acquisition to the safety cue}

To further test whether the Safety Cue can be considered a conditioned inhibitor, we conducted an additional test. If the Safety Cue has taken on inhibitory properties through conditioned inhibition, then pairing the learned Safety Cue with footshock should result in slower fear learning to the Safety Cue (Rescorla, $1969 b)$. A separate group of rats $(n=8)$ underwent reward, fear, and safety training (see Materials and Methods). A session of fear conditioning only was conducted after the reward training and the habituation session. During this session, conditioned freezing to the Fear Cue developed (one-way repeated-measures ANOVA; main effect of trial, $F_{(3,21)}=7.111 ; p<0.01$; Fig. 2, left panel), and cue-evoked freezing during the test trial one day after Fear Cue training was greater than that observed during the first acquisition trial (planned comparison, paired $t$ test, $t=-7.05, p<$ 0.001 ), but not the last acquisition trial (planned comparison, paired $t$ test, $t=-0.92, p>0.05)$. Safety conditioning followed for four sessions. One day after the last safety session, the Safety Cue was paired with a footshock four times (Fig. 2, right panel).
Conditioned freezing did not develop over these four trials (oneway repeated-measures ANOVA; $\left.F_{\operatorname{trial}(3,21)}=1.531 ; p>0.05\right)$. Freezing to the Safety Cue was reassessed $1 \mathrm{~d}$ later, and cueevoked freezing still did not significantly differ from the first acquisition trial (planned comparison, paired $t$ test, $t=-1.66$, $p>0.05$ ), meaning that conditioned freezing to the Safety Cue did not develop after pairing the Safety Cue with footshock. This indicates that the Safety Cue is a conditioned inhibitor; i.e., the Safety Cue is associated with the absence of footshock.

\section{Distinct BA neuronal populations change their firing rate} during presentation of either the Fear or Fear + Safety Cues A total of 112 single neurons located in the BA (Fig. 3A) from 14 rats were isolated from recordings made during both early (first safety session) and late (final two safety sessions) safety training (Fig. $3 B$ ). Most neurons had low mean firing rates (median = $0.83 \mathrm{~Hz}$; maximum $=20.35 \mathrm{~Hz}$; minimum $=0.06 \mathrm{~Hz}$ ), suggesting that we sampled from predominantly putative projection neurons (Likhtik et al., 2006). Each BA neuron was classified based upon its change in firing rate (permutation test, $p<0.05$ ) during the first $200 \mathrm{~ms}$ of each cue type as compared to the $20 \mathrm{~s}$ before cue presentation during late safety training, when the rats showed significant discrimination between the Fear Cue and Fear + Safety Cues (Fig. 3B). A two-way repeated-measures ANOVA on percent time spent freezing revealed a significant interaction between phase of training and cue type $\left(F_{(3,39)}=8.575, p<0.001\right)$ and a main effect of phase of training $\left(F_{(1,13)}=5.118, p<0.05\right)$ and cue type $\left(F_{(3,39)}=29.331, p<0.001\right)$. Freezing to the Fear Cue was significantly greater than the Fear + Safety Cues, Safety Cue, and Reward Cue during late safety training (post hoc Tukey's, $p<0.001$ each comparison). During late safety training, significant inhibition of firing to at least one cue within the first $200 \mathrm{~ms}$ after cue onset compared to the $20 \mathrm{~s}$ before cue presentation was observed in 49 of 112 recorded neurons, whereas a significant excitation of firing to at least one cue was seen in 29 of 112 neurons. Neurons were initially segregated into three classes (Fig. 3C). During late safety training, we found that $17.9 \%$ (20 of 112) of recorded BA neurons changed their firing rates compared to baseline in the SAME direction, i.e., both increases in firing rate $(n=7)$, or both decreases in firing rate $(n=13)$ to both the Fear and the Fear + Safety Cues. When directly comparing the response to these cues within each neuron, the magnitude of these increases and decreases did not differ between the Fear Cue and Fear + Safety Cues within the first $200 \mathrm{~ms}$ of cue onset (permutation tests, $p>0.05$ ). Of these 20 neurons, 15 neurons also showed a significant change in firing rate to the Reward Cue in the same direction as the Fear and Fear + Safety Cues. Hence, this population may represent previously described populations that encode stimulus salience rather than valence (Shabel and Janak, 2009). When we compared the responses during late safety training to early safety training, we observed that two of the 20 SAME neurons showed a significant change in firing rate to the Reward Cue, Fear Cue and Fear + Safety Cues during both early and late safety training.

Neurons without a significant change in firing rate during late safety training to either the Fear or Fear + Safety Cues were classified as NO CHANGE ( 49 of 112 recorded neurons; note that 12 of these 49 neurons did show a significant change in firing rate to the Reward Cue). Even though they did not show a significant difference compared to baseline during late safety training, 3 of these 49 NO CHANGE neurons did show a significant difference in firing rate activity when responding during the Fear Cue and the Fear + Safety Cues was directly compared (permutation tests, 
$p<0.05)$. When we compared the responses during late safety training to early safety training, we observed that 4 of the 49 NO CHANGE neurons showed a significant change in firing rate to the Reward Cue during early safety training.

In contrast, $38.4 \%$ (43 of 112) of BA neurons significantly changed their firing rate to one, but not the other cue, or showed opposite changes in firing rate to the Fear and Fear + Safety Cues and were classified as SELECTIVE. This latter finding indicates that populations of BA neurons differentially encode cues signifying fear and safety.

\section{Single BA neurons respond differentially to safety, fear, and reward cues}

Neurons classified as SELECTIVE $(n=$ 43) were further segregated into more specific subpopulations (Fig. 3D). Of the 43 neurons that showed a significant change in firing to either the Fear or Fear + Safety Cues during late safety training, $23.3 \%(n=10)$ showed a significant change in firing rate only to the Fear + Safety Cues and Safety Cue (permutation test, $p<0.05$ ) compared to the precue $20 \mathrm{~s}$ baseline. These neurons also showed differential responding, as shown by a significant difference in firing rate within the first $200 \mathrm{~ms}$ of cue presentation to the Fear + Safety Cues and Safety Cue as compared to the Fear Cue (permutation tests, $p<0.05)$. These units were termed "safety" selective. Both increases $(n=3$; Fig. $4 A 2, C)$ and decreases $(n=7$; Fig. $4 B 2$ ) in firing rate were seen in response to the Fear + Safety Cues and Safety Cue. Interestingly, these safety-selective neural responses were generally specific to late safety training sessions only (i.e., final two safety sessions). During early safety training (i.e., the first safety session), these changes in firing rate to the Fear + Safety Cues and Safety Cue were not yet evident (Fig. 4A1,B1). One neuron that showed a significant increase in firing rate to the Fear + Safety Cues and Safety Cue during late safety training also showed a significant increase in firing rate to the Safety Cue during early safety training but the magnitude of the response was significantly greater during late safety training than early safety training (permutation test, $p<0.05$ ). All other safety neurons showed no significant changes in firing rate to any cue during early safety training (permutation test, $p>0.05$ ). Thus, the increases and decreases in firing rate seen in response to the Fear + Safety Cues and Safety Cue developed over the course of learning in parallel with the rats' discrimination between the Fear and Fear + Safety Cues in late safety training (Fig. 3B).

\section{Similar change in firing to cues signifying safety and reward} In addition to safety-selective neurons, other specific subpopulations were isolated from the group of neurons classified as SE-
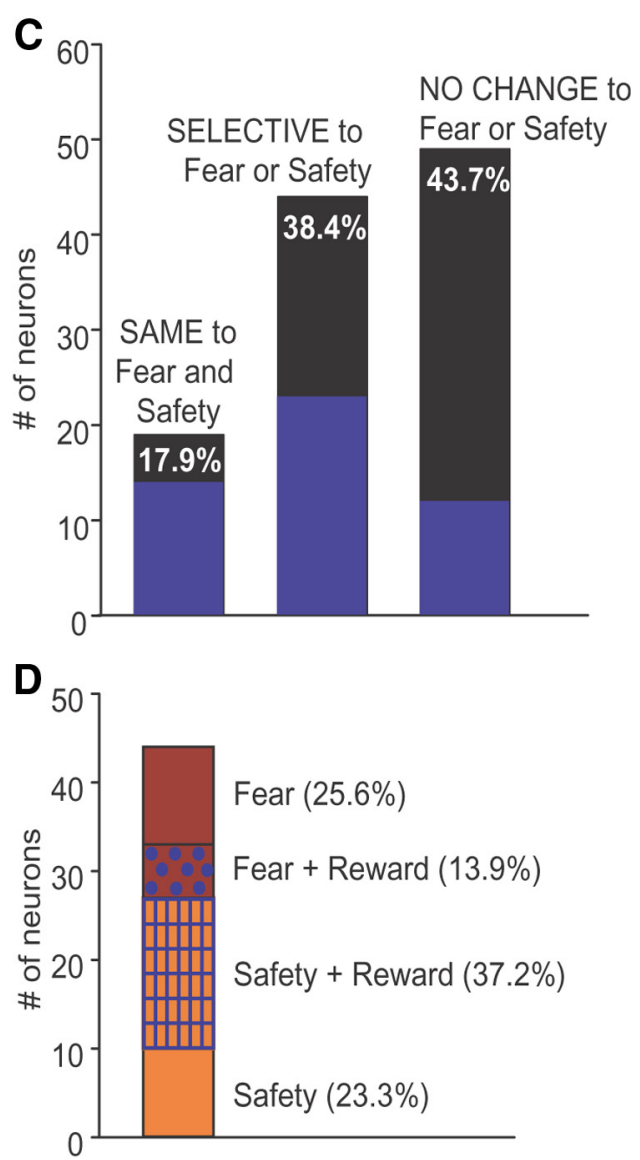

Figure 3. A, Locations of each electrode tip from 14 rats. All 112 recorded neurons were in the BA. $B$, Mean ( \pm SEM) percentage of time spent freezing during each cue during early and late safety training for the 14 rats implanted with electrode arrays. Freezing to Reward Cue, Fear + Safety Cue, and Safety Cue during late safety training. C, Neurons were initially segregated into 3 (lasses. SAME denoted significant change in firing in the same direction to the Fear and Fear + Safety Cues (17.9\% of recorded 作 blue indicate the number of neurons showing a significant change in firing rate to the Reward Cue: 15 of 20 SAME neurons showed and 12 of 49 NO CHANGE neurons were responsive to the Reward Cue. D, Neurons classified as SELECTIVE $(n=43)$ were further segregated. Safety denoted significant change in firing to only the Fear + Safety Cues and Safety Cue (23.3\% of SELECTIVE same direction (37.2\% of SELECTIVE neurons; $n=16)$. Fear and Reward denoted significant change in firing to the Fear and Reward Cues in the same direction (13.9\% of SELECTIVE neurons; $n=6$ ). Fear denoted significant change to only the Fear Cue ( $25.6 \%$ of SELECTIVE neurons; $n=11)$.

LECTIVE (Fig. 3D). Of these 43 SELECTIVE neurons, 37.2\% $(n=16)$ displayed a significant change in firing rate in the same direction to the Fear + Safety Cues, Safety Cue, and Reward Cue during late safety training (permutation test, $p<0.05$ ), but not the Fear Cue, lending support to our hypothesis that safety and reward learning may involve overlapping mechanisms. Furthermore, these neurons each showed a significant difference in firing rate within the first $200 \mathrm{~ms}$ to the Fear + Safety Cues, Safety Cue, and Reward Cue as compared to the Fear Cue, respectively (permutation tests, $p<0.05)$. Both increases $(n=11$; Fig. 4D2) and decreases $(n=5$; Fig. 4E2, $F)$ in firing rate were seen in response to the Fear + Safety Cues, Safety Cue, and Reward Cue, and these phasic responses developed over training. Specifically, these neurons did not show a significant change in firing rate to the Fear + Safety Cues or Safety Cue during early safety training (permutation test, $p>0.05$; Fig. $4 D 1, E 1$ ). In response to the Reward Cue, 
A1

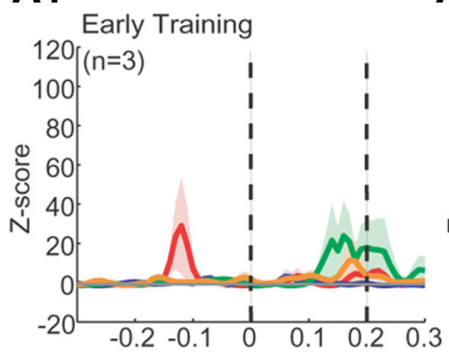

A2

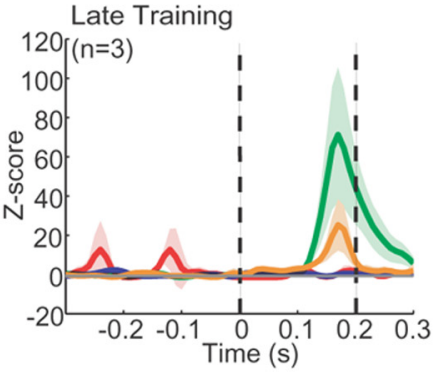

B2
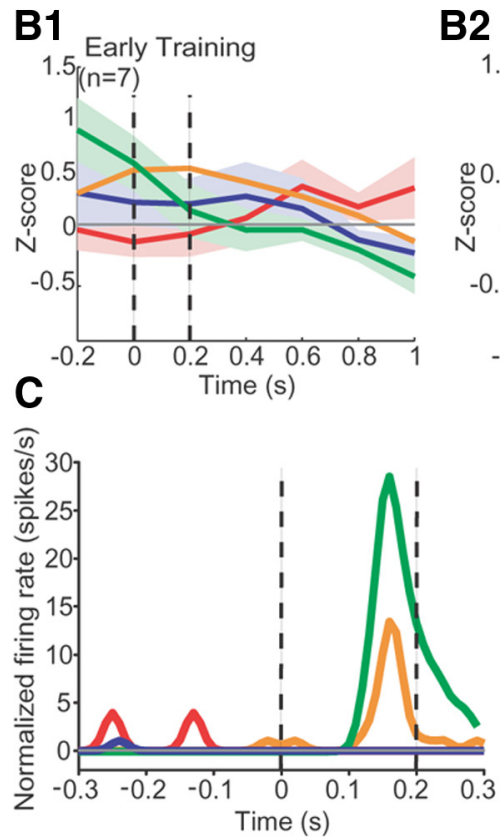

- Safety cue alone

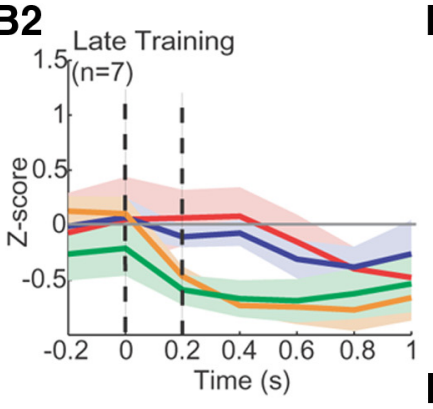

E1
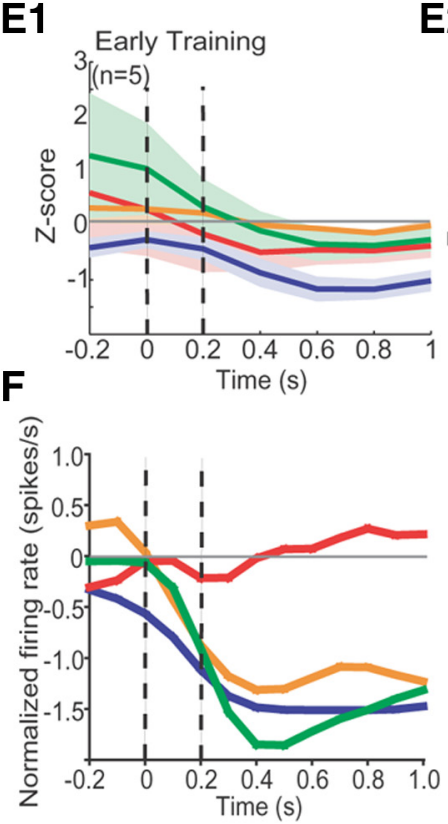

D2

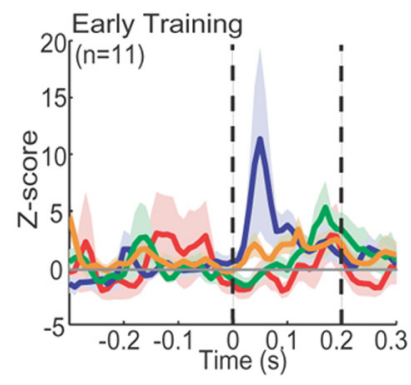

E2
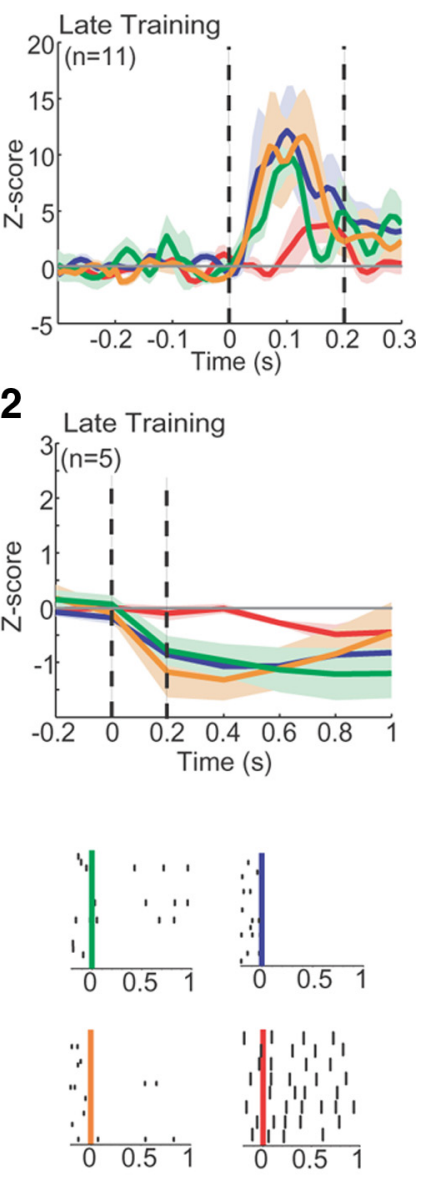

— Reward cue

Fear cue

Figure 4. BA neurons respond differentially to Safety, Fear, and Reward Cues. Peristimulus histograms (PSTHs) of firing rate activity were normalized to the $20 \mathrm{~s}$ before cue onset and smoothed with a Gaussian filter width of 3 . Vertical dashed lines indicate time window of analysis; cue onset at 0 s. Green line, activity in response to the Safety Cue alone; orange line, activity in response to the Fear + Safety Cues; blue line, activity in response to the Reward Cue; red line, activity in response to the Fear Cue. Shading indicates standard error. $A$, Population histograms of neurons $(n=$ 3) demonstrating a significant increase in firing rate to the Fear + Safety Cues and Safety Cue alone during late safety training (A2). The magnitude of this increase in firing rate increased from early safety training (A1) to late safety training (A2). PSTH bin size, $10 \mathrm{~ms}$. B, Population histograms of neurons $(n=7)$ demonstrating a significant decrease in firing rate to the Fear + Safety Cues and Safety Cue alone during late safety training (B2) that was not evident during early safety training (B1). PSTH bin size of $200 \mathrm{~ms}$. C, Example of a single BA neuron with an increase in firing activity in response to the Fear + Safety Cues and Safety Cue alone during late safety training. PSTH bin size, $10 \mathrm{~ms}$. Insets, Raster plots of same neuron with 0 s as cue onset. Each row of dots depicts action potentials on one trial. $\boldsymbol{D}$, Population histograms of neurons $(n=11)$ demonstrating a significant increase in firing rate to the Fear + Safety Cues, Safety Cue alone, and Reward Cue during late safety training (D2). This increase was not evident in response to the Fear + Safety Cues or Safety Cue alone during early safety training (D1). An increase in firing rate in response to the Reward Cue was apparent during early safety training (D1) in two neurons. PSTH bin size, $10 \mathrm{~ms}$. E, Population histograms of neurons $(n=5)$ demonstrating a significant decrease in firing rate to the Fear + Safety Cues, Safety Cue alone, and Reward Cue during late safety training (E2). This decrease was not evident during early safety training (E1). PSTH bin size, $200 \mathrm{~ms}$. $\boldsymbol{F}$, Example of a single BA neuron with a decrease in firing activity in response to the Reward Cue, Fear + Safety Cues, and Safety Cue alone during late safety training. PSTH bin size, 200 ms. Insets, Raster plots of same neuron with 0 s as cue onset. Each row of dots depicts action potentials on one trial.

however, two neurons did show a significant increase in firing rate during early safety training (permutation test, $p<0.05$; Fig. $4 D 1)$. Since rats were pretrained on the Reward Cue-sucrose pairing for seven sessions, this increase may be a reflection of that prior learning. All other safety + reward neurons showed no significant changes in firing rate to the Reward Cue during early safety training (permutation test, $p>0.05$ ). Overall, the increases and decreases in firing rate seen in response to the Fear + Safety Cues, Safety Cue, and Reward Cue developed over the course of learning.

Additionally, as expected, a subset of neurons showed a significant change in firing to the Fear Cue $(n=11,25.6 \%)$ alone. A small number of neurons showing a significant change in firing in the same direction to both the Fear and Reward Cues $(n=6$, $13.9 \%)$ were also observed.

\section{Discussion}

Here we have shown that BA neurons respond to sensory cues signifying safety. Specifically, we found that the neural response to the combined Fear + Safety Cue was different from the neural response to the presentation of the Fear Cue alone. Thus, the suppression of behavioral responding to the Fear Cue by the simultaneous presentation of the Safety Cue may depend upon differential encoding of the Fear Cue and the combined Fear + Safety Cue in the BA. These findings support a role for the BA in 
the acquisition and/or expression of behavioral responding to Safety Cues.

To determine the role of the BA in signaling safety, we developed a novel protocol to investigate the behavioral expression of fear, safety from fear, and reward seeking in parallel, permitting us to follow a single neuron's firing activity in response to cues that differentially guide the animal's behavior. We showed that rats learn to use a cue to significantly reduce its fear behavior, indicating that they learned the situation was "safe" in the presence of this Safety Cue. In addition, when this learned Safety Cue was later paired with a footshock, rats showed a dramatic reduction in the acquisition of freezing to the Safety Cue. Together, this indicates that the Safety Cue acted as a conditioned inhibitor, i.e., the Safety Cue came to predict the absence of footshock, thereby resulting in an attenuation of conditioned freezing.

Using this protocol, we recorded electrophysiological activity from single BA neurons in freely behaving rats and analyzed changes in firing rate in response to Fear, Fear + Safety, Safety, and Reward Cues over the course of safety learning. We identified a population of BA neurons that significantly changed their firing rate to combined Fear + Safety Cues, but not the Fear Cue alone, and other neurons that responded to the Fear Cue alone, but not to the Fear + Safety Cue. This finding suggests that the presentation of a Safety signal at the same time as the Fear Cue altered the neural processing of the Fear Cue in the BA. These findings suggest that neural processing of the Fear + Safety Cues contributed to the reduction in freezing in response to these cues, although a causal relationship remains to be determined.

Our findings extend prior work investigating the neuronal responses to safety cues (for review, Christianson et al., 2012). Notably, primates with extensive amygdala damage cannot discriminate a fear signal from a safety signal (Kazama et al., 2012), and the human amygdala can track the predictive aversive value of a stimulus as it reverses from fear to safety (Schiller et al., 2008). Rogan et al. (2005) reported that safety cues that predict the absence of footshock evoke a smaller cue-evoked field potential in the lateral nucleus of the amygdala (LA), a region that projects to the BA, after learning relative to before learning. Additionally, synapse size in the LA changes bidirectionally with fear and safety learning, with fear conditioning associated with larger synapses and safety conditioning associated with smaller synapses (Ostroff et al., 2010). Reduced ability of safety cues to drive LA neuronal activity could lead to reduced excitation of neurons efferent to the LA in structures such as the BA. There is also evidence to suggest that safety conditioning recruits projections from the insula to the BA. Insula lesions before safety conditioning attenuate the fear-reducing effects of safety signals, and safety signals themselves attenuate stress-induced c-fos activation in BA neurons (Christianson et al., 2008, Christianson et al., 2011). A potential role for areas downstream from the BA is also indicated by prior findings; in rats that underwent conditioned inhibition training, stimulation of the BA, in slice, elicits strong excitatory responses in lateral central nucleus neurons that then lead to increased inhibition of medial central nucleus neurons (Amano et al., 2010). How these reported changes relate to the unit responses we observed in the BA during within-session presentation of both Fear and Fear + Safety cues that were encoded with both excitations and inhibitions is not clear. Notably, while the presence of inhibitions in the BA has been reported in response to fear and reward cues (Muramoto et al. 1993; Ambroggi et al., 2008; Shabel and Janak, 2009; Amano et al., 2011; Shabel et al., 2011), the focus is usually upon phasic increases in spike activity. It is not clear what the source of cue-evoked inhibition, nor its downstream effects, might be. Together, the evidence suggests that safety conditioning recruits a circuit that includes the BA, but the mechanisms underlying the diversity of the neural responses we observed here requires further study.

We observed a population of BA neurons that showed a similar change (either a decrease or an increase) in firing rate in response to the Fear + Safety Cues, Safety Cue, and Reward Cue, supporting the idea that safety and reward learning share underlying mechanisms (Rescorla, 1969a; Walasek et al., 1995; Tanimoto et al., 2004; Rogan et al., 2005; Leknes et al., 2011). This is advantageous from an evolutionary perspective; when an animal perceives an environment as dangerous, reward seeking would be inhibited to focus on immediate survival. Conversely, correctly recognizing an environment devoid of immediate danger would suppress fear responses and increase appetitive behaviors, such as seeking food or a mate, thereby promoting survival. Our observed overlap in the encoding of safety and reward in the $\mathrm{BA}$ is consistent with a more general role for the BA in encoding stimulus valence (Paton et al., 2006; Belova et al., 2008; Shabel et al., 2011).

Our data indicate that learned safety behavior is correlated with parallel changes in neuronal firing in the BA. Neurons that responded to the Fear + Safety Cues and the Safety Cue, but not the Fear or Reward Cue (safety neurons), and neurons that responded to the Fear + Safety Cues, Safety Cue, and Reward Cue, but not the Fear Cue (safety + reward neurons), generally acquired these neural responses over the course of safety learning. Critically, during early safety training rats showed poor discrimination between the Fear Cue and Fear + Safety Cues compared to late safety training, where rats displayed good discrimination between these cues. Thus, safety and safety + reward neurons changed their rate of firing in response to Safety and Reward Cues in parallel to how well the rats discriminated among the cues.

Finally, as in our prior studies (Shabel and Janak, 2009; Shabel et al., 2011), we found a population of BA neurons that responded similarly to cues in a valence-independent manner. Since these neurons responded in a similar fashion to cues representing Fear, Safety, and Reward, these neurons may be part of a neuronal circuit that contributes to emotional arousal and/or attention, preparing the animal to respond to behaviorally relevant stimuli, consistent with prior findings by us and others (Muramoto et al., 1993; Belova et al., 2008; Shabel and Janak, 2009; Shabel et al., 2011). Overall, our findings are consistent with other studies identifying subpopulations of BA neurons showing a diversity of response profiles potentially reflective of both stimulus valence and stimulus salience. These data suggest the BA contains several neuronal subcircuits encoding a spectrum of emotional values. Presumably, these subcircuits have distinct patterns of neural inputs and outputs that allow given subcircuits to specify distinct behavioral outputs.

In summary, we show here that the rat BA contains neurons that change their firing rates differently in response to the Fear Cue versus Fear + Safety Cue, providing new evidence that single neurons respond selectively to a cue signifying safety. Our results, in addition to the several studies discussed here, implicate the amygdala in mediating the discrimination among cues signifying danger, safety, and reward. Interestingly, the S1 mouse strain has increased BA dendritic arborization and also shows impaired fear inhibition to safety cues (Camp et al., 2012), suggesting a link between amygdala dysmorphology and impaired fear inhibition. Impaired fear inhibition to safety cues, resulting in overgeneralized fear, is associated with PTSD (Jovanovic et al., 2010; Jovanic et al., 2012). Further exploration of the neural circuits that regu- 
late fear inhibition, including those that underlie safety learning, may lead to greater understanding of the processes that go awry in PTSD and similar disorders.

\section{References}

Amano T, Unal CT, Paré D (2010) Synaptic correlates of fear extinction in the amygdala. Nat Neurosci 13:489-494. CrossRef Medline

Amano T, Duvarci S, Popa D, Paré D (2011) The fear circuit revisited: contributions of the basal amygdala nuclei to conditioned fear. J Neurosci 31:15481-15489. CrossRef Medline

Ambroggi F, Ishikawa A, Fields HL, Nicola SM (2008) Basolateral amygdala neurons facilitate reward-seeking behavior by exciting nucleus accumbens neurons. Neuron 59:648-661. CrossRef Medline

Baxter MG, Murray EA (2002) The amygdala and reward. Nat Rev Neurosci 3:563-573. CrossRef Medline

Belova MA, Paton JJ, Salzman CD (2008) Moment-to-moment tracking of state value in the amydala. J Neurosci 28:10023-10030. CrossRef Medline

Benjamini Y, Hochberg Y (1995) Controlling the false discovery rate: a practical and powerful approach to multiple testing. J R Stat Soc Ser B $57: 289-300$

Blanchard RJ, Blanchard DC (1969) Crouching as an index of fear. J Comp Physiol Psychol 67:370-375. CrossRef Medline

Camp MC, MacPherson KP, Lederle L, Graybeal C, Gaburro S, DeBrouse LM, Ihne JL, Bravo JA, O'Connor RM, Ciocchi S, Wellman CL, Lüthi A, Cryan JF, Singewald N, Holmes A (2012) Genetic strain differences in learned fear inhibition associated with variation in neuroendocrine, autonomic, and amygdala dendritic phenotypes. Neuropsychopharmacology 37: 1534-1547. CrossRef Medline

Christianson JP, Benison AM, Jennings J, Sandsmark EK, Amat J, Kaufman RD, Baratta MV, Paul ED, Campeau S, Watkins LR, Barth DS, Maier SF (2008). J Neurosci 28:13703-13711. CrossRef

Christianson JP, Jennings JH, Ragole T, Flyer JGN, Benison AM, Barth DS, Watkins LR, Maier SF (2011) Safety signals mitigate the consequences of uncontrollable stress via a circuit involving the sensory insular cortex and bed nucleus of the stria terminalis. Biol Psychiatry 70:458-464. CrossRef Medline

Christianson JP, Fernando ABP, Kazama AM, Jovanovic T, Ostroff LE, Sangha S (2012) Inhibition of fear by learned safety signals: a minisymposium review. J Neurosci 32:14118-14124. CrossRef Medline

Davies DL, Bouldin DW (1979) IEEE Trans. Pattern Anal Machine Intell 1:224-227. CrossRef Medline

Fendt M, Fanselow MS (1999) The neuroanatomical and neurochemical basis of conditioned fear. Neurosci Biobehav Rev 23:743-760. CrossRef Medline

Herry C, Ciocchi S, Senn V, Demmou L, Müller C, Lüthi A (2008) Switching on and off fear by distinct neuronal circuits. Nature 454:600-606. CrossRef Medline

Hesterberg T, Moore DS, Monaghan S, Clipson A, Epstein R (2005) Bootstrap methods and permutation tests. In: Introduction to the practice of statistics, Ed 5 (Moore DS, McCabe GP, eds), pp 14-1-14-70. New York: Freeman.

Jackson A, Fetz EE (2007) Compact movable microwire array for long-term chronic unit recording in cerebral cortex of primates. J Neurophysiol 9:3109-3118. CrossRef Medline

Janak PH (2002) Multichannel neural ensemble recording during alcohol self administration. In: Methods in alcohol-related neuroscience research (Liu Y, Lovinger DM, eds), pp 241-258. Boca Raton, FL: CRC.

Jovanovic T, Norrholm SD, Blanding NQ, Davis M, Duncan E, Bradley B, Ressler KJ (2010) Impaired fear inhibition is a biomarker of PTSD but not depression. Depress Anxiety 27:244-251. CrossRef Medline

Jovanovic T, Kazama A, Bachevalier J, Davis M (2012) Impaired safety signal learning may be a biomarker of PTSD. Neuropharmacology 62:695-704. CrossRef Medline

Kazama AM, Heuer E, Davis M, Bachevalier J (2012) Effects of neonatal amygdala lesions on fear learning, conditioned inhibition, and extinction in adult macaques. Behav Neurosci 126:392-403. CrossRef Medline

LeDoux JE (2000) Emotion circuits in the brain. Annu Rev Neurosci 23: 155-184. CrossRef Medline

Leknes S, Lee M, Berna C, Andersson J, Tracey I (2011) Relief as a reward: hedonic and neural responses to safety from pain. PLoS One 6:e17870. CrossRef Medline

Likhtik E, Pelletier JG, Popescu AT, Paré D (2006) Identification of basolateral amygdala projection cells and interneurons using extracellular recordings. J Neurophysiol 96:3257-3265. CrossRef Medline

Muramoto K, Ono T, Nishijo H, Fukuda M (1993) Rat amygdaloid neuron responses during auditory discrimination. Neuroscience 52:621-636. CrossRef Medline

Nicolelis MA, Dimitrov D, Carmena JM, Crist R, Lehew G, Kralik JD, Wise SP (2003) Chronic, multisite, multielectrode recordings in macaque monkeys. Proc Natl Acad Sci U S A 100:11041-11046. CrossRef Medline

Ostroff LE, Cain CK, Bedont J, Monfils MH, LeDoux JE (2010) Fear and safety learning differentially affect synapse size and dendritic translation in the lateral amygdala. Proc Natl Acad Sci U S A 107:9418-9423. CrossRef Medline

Pape HC, Paré D (2010) Plastic synaptic networks of the amygdala for the acquisition, expression, and extinction of conditioned fear. Physiol Rev 90:419-463. CrossRef Medline

Paton JJ, Belova MA, Morrison SE, Salzman CD (2006) The primate amygdala represents the positive and negative value of visual stimuli during learning. Nature 439:865-870. CrossRef Medline

Paxinos G, Watson C (2007) The rat brain in stereotaxic coordinates, Ed 6. Amsterdam: Elsevier.

Pollak DD, Monje FJ, Zuckerman L, Denny CA, Drew MR, Kandel ER (2008) An animal model of a behavioral intervention for depression. Neuron 60:149-161. CrossRef Medline

Rescorla RA (1969a) Establishment of a positive reinforcer through contrast with shock. J Comp Physiol Psychol 67:260-263. CrossRef Medline

Rescorla RA (1969b) Pavlovian conditioned inhibition. Psychol Bull 72:7794. CrossRef

Rogan MT, Leon KS, Perez DL, Kandel ER (2005) Distinct neural signatures for safety and danger in the amygdala and striatum of the mouse. Neuron 46:309-320. CrossRef Medline

Salzman CD, Fusi S (2010) Emotion, cognition, and mental state representation in amygdala and prefrontal cortex. Annu Rev Neurosci 33:173-202. CrossRef Medline

Schiller D, Levy I, Niv Y, LeDoux JE, Phelps EA (2008) From fear to safety and back: reversal of fear in the human brain. J Neurosci 28:11517-11525. CrossRef

Shabel SJ, Janak PH (2009) Substantial similarity in amygdala neuronal activity during conditioned appetitive and aversive emotional arousal. Proc Natl Acad Sci U S A 106:15031-15036. CrossRef Medline

Shabel SJ, Schairer W, Donahue RJ, Powell V, Janak PH (2011) Similar neural activity during fear and disgust in the rat basolateral amygdala. PLoS One 6:e27797. CrossRef Medline

Tanimoto H, Heisenberg M, Gerber B (2004) Event timing turns punishment to reward. Nature 430:983. CrossRef Medline

van der Kolk BA (1997) The psychobiology of posttraumatic stress disorder. J Clin Psychiatry 58 [Suppl 9]:16-24. Medline

Walasek G, Wesierska M, Zielinski K (1995) Conditioning of fear and conditioning of safety in rats. Acta Neurobiol Exp (Wars) 55:121-132. Medline 